Fernández-Lasarte, O.; Goñi, E., Camino, I. y Zubeldia, M. (2019). Ajuste escolar y autoconcepto académico en lá Educación Secundaria. Revista de Investigación Educativa, 37(1), 163-179.

DOI: http://dx.doi.org/10.6018/rie.37.1.308651

\title{
Ajuste escolar y autoconcepto académico en la Educación Secundaria
}

\section{School Adjustment and Academic Self-concept in Secondary Education}

\author{
Oihane Fernández-Lasarte*, Eider Goñi**, Igor Camino**** y Miren Zubeldia**** \\ *Departamento de Didáctica y Organización Escolar. Universidad del País Vasco (España) \\ **Departamento de Psicología Evolutiva y de la Educación. Universidad del País Vasco (España) \\ ****Departamento de Teoría e Historia de la Educación. Universidad del País Vasco (España) \\ *****Conservatorio Profesional de Música Ataúlfo Argenta de Santander (España)
}

\begin{abstract}
Resumen
El ajuste escolar es un factor prioritario a considerar para comprender y dar una respuesta psicoeducativa a los crecientes problemas de fracaso escolar, absentismo y convivencia en las aulas. Por su parte, el autoconcepto juega un papel decisivo en el ajuste del alumnado. Los objetivos de este estudio son analizar la relación entre las dimensiones académica y global del autoconcepto y las escalas del ajuste escolar; examinar las diferencias en el ajuste escolar, así como en el autoconcepto académico y global, según el sexo y la edad del alumnado; y comprobar la capacidad explicativa del dominio académico y global del autoconcepto sobre el ajuste escolar. En el estudio participan 1468 estudiantes entre 12 y 17 años ( $M=14.03$; D.T. 1.36$), 51 \%$ mujeres y $49 \%$ hombres. Se emplean la Escala Breve de Ajuste Escolar EBAE-y el Cuestionario de Autoconcepto Dimensional-AUDIM-. El autoconcepto mantiene relaciones significativas con el ajuste escolar. Por otra parte, las mujeres informan de un mayor ajuste escolar y autoconcepto académico verbal, mientras que los hombres perciben un mayor autoconcepto académico matemático y global. Además, el autoconcepto y el ajuste escolar son mayores en la adolescencia temprana que en la adolescencia media. Por último,
\end{abstract}

Correspondencia: Oihane Fernández-Lasarte, oihane.fernandezl@ehu.eus, Departamento de Didáctica y Organización Escolar. Universidad del País Vasco. 
el autoconcepto académico y global tienen una capacidad predictiva grande-moderada sobre las dimensiones del ajuste escolar, siendo destacable la influencia del autoconcepto académico sobre el rendimiento escolar.

Palabras clave: ajuste escolar; rendimiento escolar; autoconcepto académico; educación secundaria.

\section{Abstract}

School Adjustment is considered to be an outstanding factor in order to understand and give a pshycoeducational response to the growing problems of academic failure, truancy and cohabitation in the classroom. Self-concept has likewise an essential role in students' adjustment. The aims of this study are to analyse the relationship among Academic and Global dimensions of Self-concept and School Adjustment's scales; to examine the differences in School Adjustment, as well as in Academic and Global Self-concept, according to the students' gender and age; and to check predictive ability of Academic and Global Self-concept on School Adjustment. 1468 students participated in this studybetween 11 and 17 years of age $(M=14.03$; $S D=1.36), 51 \%$ women and 49\% men. The Brief Scale of School Adjustment (EBAE) and the Multidimensional Self-Concept Questionnaire (AUDIM) were implemented. The School Adjustment keeps significant relationships with the Self-concept. Furthermore, women have been considered to have a larger School Adjustment and Academic Self-concept in oral skills, while men perceive better the Mathematic and Global Self-concept. Besides, the Self-concept and the School Adjustment are larger in the early adolescence than in the middle adolescence. Finally, the Academic and Global Self-concept have a high and moderate predictive ability about the School Adjustment's dimensions, and the Academic Self-concept a remarkable influence regarding the academic achievement.

Keywords: school adjustment; school performance; academic self-concept; secondary education.

\section{Introducción}

El ajuste escolar es definido como la competencia que necesita el alumnado para afrontar las tareas escolares exitosamente o como su capacidad de adaptación a las demandas cognitivas e interpersonales de la escuela (Ladd, 1989; Rodríguez-Fernández, Ramos-Díaz, Madariaga, Arrivillaga \& Galende, 2016). El ajuste escolar representa el grado en el que el alumnado se percibe satisfecho, integrado y comprometido con la escuela (Azpiazu, Esnaola \& Ros, 2014). Si bien en la literatura científica no existe acuerdo en su conceptualización, se acepta su estructura multidimensional. El alumnado muestra su ajuste escolar a través del rendimiento académico, las expectativas de continuar con los estudios y el comportamiento con los iguales y el profesorado (Moral de la Rubia, Sánchez-Sosa \& Villarreal-González, 2010; Rodríguez-Fernández, Droguett \& Revuelta, 2012). Las expectativas académicas se relacionan con el ajuste escolar y el logro académico (Kiuru, Aunola, Vuori \& Nurmi, 2007), y las relaciones del alumnado con los iguales y el profesorado influyen en el ajuste escolar (Ladd, Kochenderfer-Ladd \& Rydell, 2011). Por tanto, se deben favorecer el rendimiento, las expectativas y la calidad de las relaciones entre el alumnado y el profesorado 
para erradicar el abandono escolar (Cava, Povedano, Buelga \& Musitu, 2015). En el rendimiento académico influyen factores socio-contextuales y personales como el autoconcepto (Lee \& Shute, 2010).

El autoconcepto es una variable crucial en el ajuste del alumnado, por lo que alcanzar un autoconcepto positivo, o que el alumnado logre una imagen equilibrada de su propia persona, debe ser un objetivo prioritario de la educación y los programas de intervención (Esnaola, Goñi \& Madariaga, 2008). La revisión científica muestra la imprecisión en su definición. El autoconcepto es definido formalmente en la década de los 70 como "la percepción que una persona tiene sobre sí misma, formada a partir de las experiencias y relaciones con el medio, donde juegan un papel importante los refuerzos ambientales y los otros significativos" (Shavelson, Hubner \& Stanton, 1976, p. 411). Asimismo, en el modelo de Shavelson et al. (1976) se acepta la naturaleza multidimensional y jerárquica del autoconcepto: el autoconcepto general en la cúspide y en un nivel inferior el autoconcepto académico y el autoconcepto no académico -emocional, social y físico-. En la actualidad, el modelo de autoconcepto académico más aceptado incluye el autoconcepto matemático y el autoconcepto verbal (Marsh, 1986, 1990; Marsh et al., 2014, 2015; Marsh, Byrne \& Shavelson, 1988; Marsh, Smith \& Barnes, 1985).

En la investigación científica se asumen las influencias de variables psicológicas como el autoconcepto en el éxito escolar y el rendimiento académico (Stankov \& Lee, 2014). Existe evidencia empírica de que a mayor autoconcepto académico mayor logro académico (Marsh \& Martin, 2011). El autoconcepto general tiene capacidad explicativa sobre el ajuste escolar, pero destaca la mayor influencia de la dimensión académica del autoconcepto (Antonio-Agirre, Azpiazu, Esnaola \& Sarasa, 2015; Huang, 2011). Diversos estudios informan del poder predictivo del autoconcepto académico sobre el ajuste escolar (Rodríguez-Fernández, Droguett \& Revuelta, 2012) y el rendimiento académico (Costa \& Tabernero, 2012; Guay, Ratelle, Roy \& Litalien, 2010; SuárezÁlvarez, Fernández-Alonso \& Muñiz, 2014). En cuanto a las expectativas académicas, existe investigación que muestra su efecto sobre el logro educativo y su relación con el autoconcepto académico (Mortimer, Zhang, Wu, Hussemann \& Johnson, 2017). Respecto a los problemas en la escuela, se relacionan de forma negativa con el autoconcepto global y académico, y el autoconcepto global tiene capacidad predictiva sobre ellos (Garaigordobil, Durá \& Pérez, 2005). Se observa un menor número de estudios sobre las expectativas académicas y los problemas de integración escolar.

Hay evidencias de que las chicas muestran mayor ajuste en la escuela: rendimiento, integración, relación con iguales y profesorado, y expectativas académicas (AntonioAgirre et al., 2015; Fuentes, Alarcón, Gracia \& García, 2015; Heras \& Navarro, 2012). Las investigaciones también sugieren que el ajuste escolar y el rendimiento académico disminuyen en la adolescencia media (Antonio-Agirre et al., 2015; Hernando, Oliva \& Pertegal, 2013; Ibabe, 2016). No obstante, existen investigaciones con resultados no concluyentes respecto a las expectativas académicas y los problemas de integración: o bien no existen diferencias significativas en función de la edad (Antonio-Agirre et al., 2015), o bien la conducta disruptiva aumenta en la educación secundaria (Fuentes et al., 2015).

Igualmente, los resultados son dispares respecto al autococoncepto en función del sexo durante la adolescencia. Por un lado, el autoconcepto general podría ser mayor 
en los chicos (Rodríguez-Fernández, Ramos-Díaz, Ros, Fernández-Zabala \& Revuelta, 2016). Asimismo, existen diferencias sexuales en la educación secundaria relacionadas con un mayor logro y autoconcepto académico matemático por parte de los chicos, así como un logro y autoconcepto académico verbal superior en las chicas (FernándezZabala, Goñi, Rodriguez-Fernández \& Goñi, 2015; Vicent et al., 2015). Sin embargo, no siempre se encuentran diferencias significativas en función del sexo en cuanto al autoconcepto global (Garaigordobil, Cruz \& Pérez, 2003; Garaigordobil et al., 2005) y el autoconcepto académico (Costa \& Tabernero, 2012; Garaigordobil et al., 2003; IbarraAguirre \& Jacobo-García, 2016; Vicent et al., 2015), o las diferencias son favorables a las mujeres (Fuentes et al., 2015; Garaigordobil et al., 2005; Malo, Bataller, Casas, Gras \& González, 2011; Matalinares et al., 2005) o a los hombres (García \& Musitu, 2009). Además, el autoconcepto general y académico disminuyen en la adolescencia media con respecto a la adolescencia temprana (Ramos-Díaz, Rodríguez-Fernández, Ros \& Antonio-Agirre, 2017; Vicent et al., 2015) y se recuperan en la adolescencia tardía (Ibarra-Aguirre \& Jacobo-García, 2016).

\section{Objetivos e hipótesis}

Este estudio tiene tres objetivos: 1) analizar las relaciones entre el autoconcepto -académico y global-y las dimensiones del ajuste escolar -rendimiento escolar, expectativas académicas, problemas de integración y ajuste escolar global-; 2) comprobar si existen diferencias en el autoconcepto y el ajuste escolar en función del sexo y la edad; 3) examinar la capacidad predictiva del autoconcepto sobre el ajuste escolar.

Se formulan las siguientes hipótesis: a) hay relaciones significativas entre las dimensiones del autoconcepto y las escalas del ajuste escolar; b) existen diferencias en el autoconcepto académico y global, así como en el ajuste escolar del alumnado adolescente en función del sexo y la edad; c) el autoconcepto académico y global predicen el ajuste escolar.

\section{Método}

\section{Participantes}

En la investigación participan 1468 estudiantes (49\% hombres y 51\% mujeres) con edades comprendidas entre 12 y 17 años $(M=14.03$; $D T=1.36)$, pertenecientes a 9 centros de Educación Secundaria de la Comunidad Autónoma del País Vasco (CAPV), seleccionados aleatoriamente, entre los cuales 5 son públicos y 4 privados concertados. El alumnado se divide en dos grupos: 12-14 años (60\%), adolescencia temprana, y 15-17 años (40\%), adolescencia media. Los valores perdidos así como los outliers son eliminados.

\section{Instrumentos}

El Cuestionario de Autoconcepto Dimensional (AUDIM) (Fernández-Zabala et al., 2015) tiene 33 ítems y 5 opciones de respuesta (de $1=$ falso a $5=$ verdadero) para medir 5 escalas: 
- Autoconcepto general (5 ítems). Es la autopercepción global de la persona. Incluye ítems como "me siento feliz" o "me siento una persona afortunada".

- Autoconcepto físico (8 ítems). Es la autopercepción de las habilidades y cualidades para la actividad física y el deporte, la condición y la forma física, la apariencia física y la satisfacción con la propia imagen corporal, y la fuerza física.

- Autoconcepto personal (8 ítems). Es la percepción de la propia integridad como persona en la que se puede confiar, la estabilidad emocional, la independencia de las otras personas y la autorrealización o el logro de metas.

- Autoconcepto social (4 ítems). Es la percepción de la contribución personal al orden social establecido y las propias capacidades en las situaciones sociales.

- Autoconcepto académico (8 ítems). Es la percepción del sujeto como estudiante en las asignaturas relacionadas con lo verbal y lo matemático.

Para analizar el autoconcepto, en esta investigación se emplean la dimensión académica -general, verbal y matemática- y la medida global, la puntuación media de las 5 escalas.

El AUDIM presenta una consistencia interna con los sujetos de este estudio para todos los ítems de $\alpha=.85$, así como para los ítems del autoconcepto académico verbal de $\alpha=.81$, autoconcepto académico matemático de $\alpha=.79 \mathrm{y}$ autoconcepto académico de $\alpha=.77$. Las puntuaciones originales en la validación del cuestionario respecto a los ítems de autoconcepto académico verbal y matemático oscilan entre .77 y .79, y el alfa de la escala total es .77.

Para evaluar el ajuste escolar se utiliza la Escala Breve de Ajuste Escolar (EBAE-10) (Moral de la Rubia et al., 2010). Este instrumento presenta 10 ítems con formato Likert de 6 rangos de respuesta (desde $1=$ completamente en desacuerdo hasta $6=$ completamente de acuerdo) para evaluar tres dimensiones:

- Rendimiento escolar (3 ítems). Mide la autoeficacia percibida como buen estudiante, la motivación intrínseca en la realización del trabajo escolar junto con los buenos resultados académicos.

- Expectativas académicas (2 ítems). Se refieren a la planificación y el interés de la persona en continuar con sus estudios.

- Problemas de integración en la escuela (5 ítems). Son situaciones que implican riesgo de inadaptación escolar, debido a dificultades con el profesorado y el alumnado, como burlas y rechazo, así como por aburrimiento.

Además, el instrumento de medida permite obtener una puntuación para el ajuste escolar global percibido. En este estudio se examinan las tres dimensiones y la medida global.

La fiabilidad en la versión de validación de la escala es: $\alpha=.85$ en expectativas académicas, $\alpha=.84$ en problemas de integración, $\alpha=.78$ en rendimiento escolar y $\alpha=.79$ en la escala total. La consistencia obtenida con los datos de este estudio es: $\alpha=.75$ en rendimiento escolar, $\alpha=.47$ en expectativas académicas, $\alpha=.63$ en problemas de integración y $\alpha=.72$ en ajuste escolar global. 


\section{Procedimiento}

Los participantes son seleccionados por muestreo aleatorio estratificado, es decir, se eligen al azar algunos centros del listado oficial de la Consejería de Educación, así como los grupos de edad en cada uno de ellos. Se contacta con los centros para presentar la investigación al equipo de dirección, orientación y profesorado y se les solicita su participación voluntaria, acordando comunicarles los resultados al finalizar el estudio. Una vez obtenida la aprobación por parte del centro, se pide el consentimiento por escrito a las familias del alumnado menor de edad. Las investigadoras se encargan de administrar la batería de cuestionarios en formato papel, una vez obtenido el permiso de los progenitores o tutores/as, quienes reciben la explicación de la investigación vía carta. En todos los casos se solicita la participación voluntaria por parte del alumnado y se garantiza el anonimato para conseguir la sinceridad en las respuestas. Además, se sigue el procedimiento de ciego único, a través del cual los sujetos desconocen los objetivos del estudio para disminuir las expectativas, la reactividad y la deseabilidad social. Asimismo, la administración simultánea a toda una misma clase en horario lectivo pretende asegurar la homogeneidad e igualdad de condiciones. Las investigadoras están presentes durante la cumplimentación del dossier de cuestionarios para aclarar las dudas al alumnado y asegurar la total cumplimentación de las pruebas. El periodo de tiempo estimado no supera los 30 minutos para evitar el cansancio del alumnado participante.

\section{Análisis de datos}

Este estudio es de tipo comparativo, su diseño es transversal, es decir, en él se utilizan participantes de una misma cultura que pertenecen a distintos grupos naturales y son evaluados en un momento temporal concreto en más de una variable dependiente (Ato, López \& Benavente, 2013).

Los análisis estadísticos se realizan mediante el programa SPSS versión 24. Primeramente, se comprueban los supuestos de normalidad y homocedasticidad del modelo lineal general: la mayoría de las escalas incumplen la normalidad y existen grupos heterogéneos. No obstante, los histogramas permiten comprobar el ajuste de los puntos al eje de normalidad y los índices de asimetría y curtosis reflejan que la mayoría de las variables no exceden el valor $|1|$ e incluso las dimensiones que lo exceden no suponen una asimetría grave | 2 | ni una curtosis severa |3| (Chok, 2010; West, Finch \& Curran, 1995). Por consiguiente, se decide emplear pruebas paramétricas, puesto que son pruebas robustas aún cuando no existe violación grave de los supuestos (Chok, 2010; Montilla \& Kromrey, 2010). Además, los niveles de tolerancia y los factores de inflación de varianza garantizan la ausencia de colinealidad entre las variables regresoras. Asimismo, es posible asumir la normalidad y la homocedasticidad de los residuos mediante la inspección visual de los histogramas, los gráficos P-P normal y los diagramas de dispersión, así como a través de la prueba Kolmogorov-Smirnov. Por todo ello se opta por emplear el análisis de regresión.

En primer lugar, se procede con las correlaciones bivariadas de Pearson, que permiten comprobar si la asociación lineal entre las dimensiones de las variables 
autoconcepto y ajuste escolar es estadísticamente significativa, así como su fortaleza y dirección. A continuación, los estadísticos descriptivos de comparación de medias, concretamente la prueba $t$ de student permite analizar si existen diferencias estadísticamente significativas en las variables objeto de estudio, esto es, en el autoconcepto -académico y global-y en el ajuste escolar -rendimiento escolar, expectativas académicas, problemas de integración y ajuste escolar global- en función del sexo y la edad. Finalmente, la regresión lineal múltiple, método de pasos sucesivos, proporciona información sobre la dependencia entre las variables, es decir, en qué medida las dimensiones del ajuste escolar pueden estar explicadas por las dimensiones del autoconcepto.

\section{Resultados}

\section{Relaciones entre el autoconcepto académico y global con el ajuste escolar}

Los coeficientes de correlación de Pearson del autoconcepto global y académico -verbal y matemático- con el ajuste escolar -rendimiento académico, expectativas académicas, problemas de integración y ajuste escolar global-, así como la fiabilidad de cada factor son mostrados en la tabla 1.

Tabla 1

Correlaciones bivariadas entre las variables y alphas de cada factor

\begin{tabular}{lcccccccc}
\hline Variable & 1 & 2 & 3 & 4 & 5 & 6 & 7 & 8 \\
\hline $\begin{array}{l}\text { 1. Autoconcepto académico } \\
\text { 2. Autoconcepto académico }\end{array}$ & - & & & & & & & \\
verbal & $.769^{*}$ & - & & & & & \\
3. Autoconcepto académico & & & & & & & \\
matemático & $.807^{*}$ & $.243^{*}$ & - & & & & \\
4. Autoconcepto global & $.686^{*}$ & $.492^{*}$ & $.586^{*}$ & - & & & \\
5. Rendimiento escolar & $.641^{*}$ & $.536^{*}$ & $.477^{*}$ & $.490^{*}$ & - & & \\
6. Expectativas académicas & $.290^{*}$ & $.273^{*}$ & $.188^{*}$ & $.217^{*}$ & $.361^{*}$ & - & & \\
7. Problemas de integración & $-261^{*}$ & $-241^{*}$ & $-.173^{*}$ & $-.330^{*}$ & $-.303^{*}$ & $-.178^{*}$ & - & \\
8. Ajuste escolar global & $.550^{*}$ & $.482^{*}$ & $.389^{*}$ & $.495^{*}$ & $.770^{*}$ & $.584^{*}$ & $-.777^{*}$ & - \\
Alpha de Cronbach & .77 & .81 & .79 & .85 & .75 & .47 & .63 & .72 \\
\hline
\end{tabular}

Nota: $N=1468 ; g l=N-2$.

${ }^{*} p<.01$.

Los coeficientes de correlación de Pearson sugieren que todas las asociaciones entre las dimensiones del autoconcepto y el ajuste escolar son estadísticamente significativas y positivas, excepto las de la escala problemas de integración, que son negativas. Por tanto, a mayor autoconcepto académico y global, mayores rendimiento escolar, expectativas 
académicas y ajuste escolar global, y menores problemas de integración; y viceversa, a menor autoconcepto, menores rendimiento escolar, expectativas académicas y ajuste escolar global, y mayores problemas de integración escolar. Respecto a la intensidad, destacan las conexiones con mayor fuerza entre: el autoconcepto académico -general, verbal y matemático- con el rendimiento escolar y el ajuste escolar global, y el autoconcepto global con el ajuste escolar global y el rendimiento escolar.

\section{Autoconcepto académico, autoconcepto global y ajuste escolar: diferencias en fun- ción del sexo y la edad}

Con el propósito de estudiar las diferencias del autoconcepto y el ajuste escolar en función del sexo y la edad, se realiza la prueba $t$ de student para muestras independientes, cuyos resultados se presentan en las tablas 2 y 3 .

Tabla 2

Autoconcepto académico y global en función del sexo y la edad

\begin{tabular}{|c|c|c|c|c|c|c|c|}
\hline Dimensiones & Sexo & $n$ & $M$ & $D T$ & $t$ & $p$ & $d_{\text {Cohen }}$ \\
\hline $\begin{array}{l}\text { Autoconcepto } \\
\text { Académico }\end{array}$ & $\begin{array}{l}\text { Hombre } \\
\text { Mujer }\end{array}$ & $\begin{array}{l}710 \\
753\end{array}$ & $\begin{array}{l}3.56 \\
3.43\end{array}$ & $\begin{array}{l}0.74 \\
0.77\end{array}$ & 3.26 & $.001^{* * *}$ & 0.170 \\
\hline $\begin{array}{l}\text { Autoconcepto } \\
\text { académico verbal }\end{array}$ & $\begin{array}{l}\text { Hombre } \\
\text { Mujer }\end{array}$ & $\begin{array}{l}710 \\
753\end{array}$ & $\begin{array}{l}3.45 \\
3.56\end{array}$ & $\begin{array}{l}0.93 \\
0.91\end{array}$ & -2.24 & $.025^{*}$ & 0.117 \\
\hline $\begin{array}{l}\text { Autoconcepto } \\
\text { académico matemático }\end{array}$ & $\begin{array}{l}\text { Hombre } \\
\text { Mujer }\end{array}$ & $\begin{array}{l}710 \\
753\end{array}$ & $\begin{array}{l}3.67 \\
3.30\end{array}$ & $\begin{array}{l}0.91 \\
1.04\end{array}$ & 7.14 & $.000^{* * *}$ & 0.375 \\
\hline $\begin{array}{l}\text { Autoconcepto } \\
\text { global }\end{array}$ & $\begin{array}{l}\text { Hombre } \\
\text { Mujer }\end{array}$ & $\begin{array}{l}710 \\
753\end{array}$ & $\begin{array}{l}3.71 \\
3.47\end{array}$ & $\begin{array}{l}0.42 \\
0.45\end{array}$ & 10.24 & $.000^{* * *}$ & 0.535 \\
\hline Dimensiones & Edad & $n$ & $M$ & $D T$ & $t$ & $p$ & $d_{\text {Cohen }}$ \\
\hline $\begin{array}{l}\text { Autoconcepto } \\
\text { académico }\end{array}$ & $\begin{array}{l}12-14 \\
15-17\end{array}$ & $\begin{array}{l}882 \\
578\end{array}$ & $\begin{array}{l}3.59 \\
3.34\end{array}$ & $\begin{array}{l}0.76 \\
0.72\end{array}$ & 6.22 & $.000^{* * *}$ & 0.348 \\
\hline $\begin{array}{l}\text { Autoconcepto } \\
\text { académico verbal }\end{array}$ & $\begin{array}{l}12-14 \\
15-17\end{array}$ & $\begin{array}{l}882 \\
578\end{array}$ & $\begin{array}{l}3.58 \\
3.39\end{array}$ & $\begin{array}{l}0.90 \\
0.94\end{array}$ & 3.75 & $.000^{* * *}$ & 0.196 \\
\hline $\begin{array}{l}\text { Autoconcepto } \\
\text { académico matemático }\end{array}$ & $\begin{array}{l}12-14 \\
15-17\end{array}$ & $\begin{array}{l}882 \\
578\end{array}$ & $\begin{array}{l}3.60 \\
3.29\end{array}$ & $\begin{array}{l}0.92 \\
1.08\end{array}$ & 5.65 & $.000^{* * *}$ & 0.342 \\
\hline $\begin{array}{l}\text { Autoconcepto } \\
\text { global }\end{array}$ & $\begin{array}{l}12-14 \\
15-17\end{array}$ & $\begin{array}{l}882 \\
578\end{array}$ & $\begin{array}{l}3.62 \\
3.53\end{array}$ & $\begin{array}{l}0.45 \\
0.45\end{array}$ & 3.91 & $.000^{* * *}$ & 0.205 \\
\hline
\end{tabular}

${ }^{*} p<.05 .{ }^{* *} p<.01 .{ }^{* * *} p<.001$.

Los resultados muestran diferencias estadísticamente significativas a favor de las mujeres únicamente en el autoconcepto académico verbal. En el resto de dimensiones del autoconcepto -global, académica y académica matemática- las puntuaciones son mayores en los hombres. Además, los análisis revelan que la diferencia de medias alcanza la significación estadística en todas las escalas del autoconcepto -global, aca- 
démica, académica verbal y académica matemática- a favor de la adolescencia temprana, que comprende los 12-14 años. El tamaño del efecto es pequeño, excepto en el autoconcepto global en función del sexo, donde es moderado.

Tabla 3

Ajuste escolar en función del sexo y la edad

\begin{tabular}{|c|c|c|c|c|c|c|c|}
\hline Dimensiones & Sexo & $N$ & $M$ & $D T$ & $t$ & $p$ & $d_{\text {Cohen }}$ \\
\hline $\begin{array}{l}\text { Rendimiento } \\
\text { escolar }\end{array}$ & $\begin{array}{l}\text { Hombre } \\
\text { Mujer }\end{array}$ & $\begin{array}{l}710 \\
753\end{array}$ & $\begin{array}{l}11.49 \\
12.09\end{array}$ & $\begin{array}{l}3.17 \\
3.11\end{array}$ & -3.63 & $.000^{* * *}$ & 0.190 \\
\hline $\begin{array}{l}\text { Expectativas } \\
\text { académicas }\end{array}$ & $\begin{array}{l}\text { Hombre } \\
\text { Mujer }\end{array}$ & $\begin{array}{l}710 \\
753\end{array}$ & $\begin{array}{l}10.20 \\
10.80\end{array}$ & $\begin{array}{l}2.13 \\
1.83\end{array}$ & -5.85 & $.000^{* * *}$ & 0.313 \\
\hline $\begin{array}{l}\text { Problemas } \\
\text { de integración }\end{array}$ & $\begin{array}{l}\text { Hombre } \\
\text { Mujer }\end{array}$ & $\begin{array}{l}710 \\
753\end{array}$ & $\begin{array}{c}10.33 \\
9.54\end{array}$ & $\begin{array}{l}3.80 \\
3.66\end{array}$ & 4.02 & $.000^{* * *}$ & 0.210 \\
\hline $\begin{array}{l}\text { Ajuste escolar } \\
\text { global }\end{array}$ & $\begin{array}{l}\text { Hombre } \\
\text { Mujer }\end{array}$ & $\begin{array}{l}709 \\
753 \\
\end{array}$ & $\begin{array}{l}46.36 \\
48.35\end{array}$ & $\begin{array}{l}6.65 \\
6.24 \\
\end{array}$ & -5.88 & $.000^{* * *}$ & 0.310 \\
\hline Dimensiones & Edad & $N$ & $M$ & $D T$ & $t$ & $p$ & $d_{\text {Cohen }}$ \\
\hline $\begin{array}{l}\text { Rendimiento } \\
\text { escolar }\end{array}$ & $\begin{array}{l}12-14 \\
15-17\end{array}$ & $\begin{array}{l}882 \\
578\end{array}$ & $\begin{array}{l}12.28 \\
11.06\end{array}$ & $\begin{array}{l}3.09 \\
3.10\end{array}$ & 7.41 & $.000^{* * *}$ & 0.388 \\
\hline $\begin{array}{l}\text { Expectativas } \\
\text { académicas }\end{array}$ & $\begin{array}{l}12-14 \\
15-17\end{array}$ & $\begin{array}{l}882 \\
578\end{array}$ & $\begin{array}{l}10.51 \\
10.51\end{array}$ & $\begin{array}{l}2.01 \\
1.98\end{array}$ & 0.07 & .942 & 0.004 \\
\hline $\begin{array}{l}\text { Problemas } \\
\text { de integración }\end{array}$ & $\begin{array}{l}12-14 \\
15-17\end{array}$ & $\begin{array}{l}882 \\
578\end{array}$ & $\begin{array}{l}9.97 \\
9.82\end{array}$ & $\begin{array}{l}3.84 \\
3.59\end{array}$ & 0.78 & .434 & 0.043 \\
\hline $\begin{array}{l}\text { Ajuste escolar } \\
\text { global }\end{array}$ & $\begin{array}{l}12-14 \\
15-17\end{array}$ & $\begin{array}{l}881 \\
578\end{array}$ & $\begin{array}{l}47.83 \\
46.75\end{array}$ & $\begin{array}{l}6.57 \\
6.34\end{array}$ & 3.13 & $.002^{* *}$ & 0.174 \\
\hline
\end{tabular}

${ }^{*} p<.05 .{ }^{* *} p<.01 .{ }^{* * *} p<.001$.

La tabla 3 evidencia la existencia de diferencias significativas entre ambos sexos favorables a las mujeres en todas las escalas del ajuste escolar: mayor rendimiento escolar, expectativas académicas y ajuste escolar global, y menores problemas de integración. Por otro lado, las diferencias son estadísticamente significativas a favor de la adolescencia temprana, representada por el grupo entre 12 y 14 años, en las escalas de rendimiento y ajuste escolar global. Sin embargo, en las escalas de expectativas académicas y problemas de integración escolar las diferencias en función de la edad no resultan estadísticamente significativas. Por último, el tamaño del efecto es siempre pequeño.

\section{Predicción del autoconcepto académico y global sobre el ajuste escolar}

La regresión lineal múltiple permite conocer el efecto del autoconcepto académico y el autoconcepto global sobre las dimensiones del ajuste escolar (ver tabla 4). Se toman como variables explicativas las dimensiones académica general y global del autoconcepto. 
Tabla 4

Capacidad predictiva del autoconcepto académico y global sobre el ajuste escolar

\begin{tabular}{|c|c|c|c|c|c|c|}
\hline Variable predictora & $R^{2}$ & $\Delta R^{2}$ & Error típico & Constante & $\beta$ & $t$ \\
\hline \multicolumn{7}{|c|}{ Rendimiento escolar $\left(F=520.27^{* * *}\right)$} \\
\hline Autoconcepto académico & .410 & .410 & 2.42 & 2.44 & .575 & $20.96^{* * *}$ \\
\hline Autoconcepto global & .415 & .414 & 2.41 & .99 & .096 & $3.49^{* * *}$ \\
\hline \multicolumn{7}{|c|}{ Expectativas académicas $\left(F=134.91^{* * *}\right)$} \\
\hline Autoconcepto académico & .084 & .084 & 1.91 & 7.82 & .290 & $11.62^{* * *}$ \\
\hline \multicolumn{7}{|c|}{ Problemas de integración $\left(F=179.15^{* * *}\right)$} \\
\hline Autoconcepto global & .109 & .108 & 3.54 & 19.75 & -.330 & $13.38^{* * *}$ \\
\hline \multicolumn{7}{|c|}{ Ajuste escolar global $\left(F=357.57^{* * *}\right)$} \\
\hline Autoconcepto académico & .302 & .302 & 5.44 & 30.81 & .397 & $13.48^{* * *}$ \\
\hline Autoconcepto global & .328 & .327 & 5.34 & 23.92 & .222 & $7.54^{* * *}$ \\
\hline
\end{tabular}

${ }^{*} p<.05 .{ }^{* *} p<.01 .{ }^{* * *} p<.001$.

En el modelo de predicción del rendimiento escolar son introducidas como variables explicativas, primeramente, el autoconcepto académico y, en segundo lugar, el autoconcepto global. Asimismo, el coeficiente beta estandarizado indica que el mayor peso explicativo sobre el rendimiento lo ejerce el autoconcepto académico. Por su parte, el coeficiente de determinación ajustado informa de un porcentaje de varianza explicada grande en el rendimiento escolar, concretamente, del $41.4 \%$. Respecto a las expectativas académicas, solo el autoconcepto académico tiene capacidad predictiva, con un menor peso y un porcentaje de varianza explicada inferior de $8.4 \%$. En cuanto a los problemas de integración, la única variable predictora es el autoconcepto global, con un peso mayor que en el modelo anterior, acercándose en mayor grado a tener un poder explicativo moderado, prediciendo el $10.8 \%$ de la varianza. Por último, en el ajuste escolar global se repite el patrón del primer modelo relativo al rendimiento escolar, es decir, coinciden los factores que son introducidos en el modelo y el orden de importancia: el autoconcepto académico y el autoconcepto global. La dimensión académica del autoconcepto es nuevamente la que mayor fuerza predictiva demuestra en comparación con el autoconcepto global, y entre ambos tienen una capacidad explicativa grande del $32.7 \%$ de la varianza del ajuste escolar global. Así, los modelos del rendimiento escolar y el ajuste escolar global son efectivos, mientras que los de las expectativas académicas y los problemas de integración no son muy efectivos.

\section{Discusión y conclusiones}

Los resultados apoyan la primera y la tercera hipótesis, que formulan la existencia de relaciones significativas entre el autoconcepto y el ajuste escolar así como la capa- 
cidad predictiva del primero sobre el segundo. Las investigaciones previas sustentan la mayor correlación y la capacidad predictiva del autoconcepto académico sobre el ajuste escolar, en especial sobre el rendimiento escolar (Costa \& Tabernero, 2012; Veas, Gilar \& Miñano, 2016) y, con menor peso, sobre las expectativas académicas (Antonio-Agirre et al., 2015), mientras que, sobre los problemas de integración, es el autoconcepto global el más influyente. Los modelos predictivos del rendimiento y el ajuste escolar global resultan efectivos; sin embargo, en las expectativas académicas y los problemas de integración los modelos no son muy efectivos al igual que en otros trabajos (Antonio-Agirre et al., 2015).

Los resultados también están en consonancia prácticamente en su totalidad con la segunda hipótesis, puesto que existen diferencias estadísticamente significativas en función del sexo y la edad en el ajuste escolar y en el autoconcepto. El mayor ajuste escolar en las chicas concuerda con estudios previos que apoyan la influencia positiva de ser chica sobre el rendimiento académico (Veas et al., 2016), lo cual podría deberse al mayor tiempo que dedican al estudio y a las tareas escolares, demostrando así la relevancia del trabajo y la motivación para el logro escolar (Hernando, Oliva \& Pertegal, 2012, 2013). Asimismo, los resultados sugieren el descenso del ajuste escolar en la adolescencia (Lee \& Chung, 2016), que podría atribuirse a la etapa vulnerable que esta representa (Feldman, 2007), caracterizada por problemas de regulación del afecto y el comportamiento (Steinberg, 2005), y cambios en los hábitos saludables que afectan al rendimiento: descenso de la actividad física y el deporte, incremento del tiempo dedicado a las amistades y el consumo de sustancias (Hernando et al., 2013), y sueño retrasado debido a horas tardías al acostarse entre semana y fines de semana (Hysing, Harvey, Linton, Askeland \& Sivertsen, 2016). Además, esta disminución del rendimiento podría tener relación con la obligatoriedad de la educación durante este tramo de la adolescencia, que implica una mayor presión académica (Hernando et al., 2012). La participación en actividades de tiempo libre podría funcionar como antídoto contra el bajo rendimiento y el estrés en la escuela (Badura et al., 2016).

Sin embargo, la segunda hipótesis no se cumple del todo, al no ser significativas las diferencias en las expectativas académicas y los problemas de integración escolar en función de la edad, coincidiendo con datos previos (Antonio-Agirre et al., 2015).

La adolescencia es una etapa en la que también se resiente el autoconcepto general y todas sus dimensiones (Fernández-Zabala \& Sarasa, 2015). El deterioro del autoconcepto académico durante la educación secundaria también podría deberse al aumento de presión en las evaluaciones (Liu \& Wang, 2005). Asimismo, el presente trabajo pretende esclarecer las discrepancias observadas en el autoconcepto en función del sexo, que podrían deberse a la diversidad de edades, procedencias de las muestras e instrumentos empleados en los estudios (Garaigordobil, Pérez \& Mozaz, 2008). En esta investigación, los chicos informan de un mayor autoconcepto global, al igual que otros estudios (García \& Musitu, 2009). Además, los chicos manifiestan un autoconcepto académico general y matemático superior, mientras que las chicas perciben un mayor autoconcepto académico verbal, también en la línea de otros trabajos (Fernández-Zabala et al., 2015; Vicent et al., 2015). Es destacable la posible influencia del estereotipo sexual, que favorece y predispone a las mujeres hacia las asignaturas 
de Lengua (Costa \& Tabernero, 2012). Igualmente, el estereotipo sexual que atribuye a los hombres una mayor capacidad hacia las matemáticas podría beneficiarles $\mathrm{y}$, por contra, generar inseguridad en las mujeres en detrimento de su motivación y rendimiento hacia las áreas de matemáticas y ciencias (González-Pienda et al., 2012). En definitiva, la investigación evidencia el efecto de los estereotipos de género por parte de las familias y el profesorado sobre el autoconcepto y el rendimiento del alumnado, siendo más favorables a los chicos (Igbo, Onu \& Obiyo, 2015).

Por lo tanto, en base a los resultados de esta investigación, se plantea como implicación educativa reforzar el autoconcepto académico para mejorar el ajuste escolar y el rendimiento. A su vez, resulta necesario desarrollar programas de intervención que involucren a toda la comunidad educativa con el propósito de trabajar el estereotipo sexual, tomar conciencia de la influencia del mismo y dotar de estrategias para conseguir una sociedad más equitativa. Además, es preciso prestar atención a la adolescencia media, puesto que es un periodo evolutivo altamente susceptible al desajuste escolar. La ciencia avala la importancia de prestar atención a los cambios de etapa y centro educativo, puesto que las intervenciones educativas pueden prevenir y disminuir el impacto negativo que tiene lugar durante la transición a la educación secundaria, logrando así una menor caída del autoconcepto (Coelho, Marchante \& Jimerson, 2017). Otra aportación de este trabajo podría ser la importancia de inculcar valores de esfuerzo y trabajo, en base a que el rendimiento tiene un gran peso dentro del ajuste escolar. También resulta prioritario continuar con la investigación sobre el ajuste escolar, con el fin de ofrecer un modelo óptimo, que, además del rendimiento, integre indicadores de calidad explicativa, y arroje luz sobre su relación con las diferentes variables (Cava et al., 2015).

De hecho, entre las limitaciones de este estudio, cabe destacar la baja consistencia interna de las subescalas expectativas académicas e integración escolar por debajo de .65, valor recomendado por Nunnally (1976) como mínimamente aceptable. Podría estar en riesgo la validez interna de los resultados obtenidos en las citadas subescalas.

Además, como investigaciones futuras, se plantea la necesidad de incluir otras variables educativas, como la implicación escolar, y atender las variables contextuales, como el apoyo del profesorado, familiar y de los iguales, para avanzar en el conocimiento del ajuste escolar e intervenir educativamente con garantías de éxito. Estas intervenciones deben dar respuesta al creciente problema que afecta a los sistemas educativos y a las sociedades, el fracaso escolar y los problemas de convivencia del alumnado en las aulas (Wang \& Fredricks, 2014); procesos que son sensibles a la interacción del alumnado con el contexto, porque requieren del apoyo de toda la comunidad educativa. En este sentido, la investigación muestra que el apoyo familiar mejora el ajuste escolar (Fuentes et al., 2015), y las expectativas familiares predicen el rendimiento del alumnado (Froiland \& Davison, 2016) y disminuyen los problemas de integración (Gerard \& Booth, 2015). Del mismo modo, las relaciones positivas con los iguales favorecen el ajuste escolar (Wentzel, Russell \& Baker, 2014), al igual que las relaciones cercanas entre el profesorado y el alumnado protegen el ajuste escolar y el rendimiento durante la transición a la etapa secundaria (Longobardi, Prino, Marengo \& Settanni, 2016). 
Igualmente, al abordar en este trabajo de forma exclusiva la educación secundaria, queda pendiente investigar la adolescencia tardía para poder verificar si se recuperan el ajuste escolar y el autoconcepto, y si las diferencias de sexo y el efecto del autoconcepto académico sobre el ajuste escolar y el rendimiento se mantienen. Así, las implicaciones educativas propuestas podrían ampliarse a la educación postobligatoria.

Por último, otra limitación del estudio presente es su corte transversal, con lo cual no es posible estudiar la evolución intra-sujeto en el ajuste escolar y el autoconcepto en las diferentes edades. De ahí, la conveniencia de llevar a cabo estudios longitudinales para obtener una mayor fiabilidad en los resultados obtenidos. Resultados que serían más válidos si, además de la percepción del alumnado, tuvieran en cuenta las calificaciones de su rendimiento en las diferentes áreas junto con otras vías de recogida de información, como las entrevistas al profesorado y a las familias del alumnado.

\section{Referencias}

Antonio-Agirre, I., Azpiazu, L., Esnaola, I., \& Sarasa, M. (2015). Predictive ability of self-concept and emotional intelligence in perceived school adjustment. BordonRevista de Pedagogía, 67(4), 9-25. doi:10.13042/Bordon.2015.67401

Ato, M., López, J. J., \& Benavente, A. (2013). Un sistema de clasificación de los diseños de investigación en psicología. Anales de Psicología, 29(3), 1038-1059. doi:10.6018/ analesps.29.3.178511

Azpiazu, L., Esnaola, I., \& Ros, I. (2014). Factores contextuales y variables individuales en el ajuste escolar. International Journal of Developmental and Educational Psychology. Revista INFAD de Psicología, 6(1), 327-336. doi:10.17060/ijodaep.2014.n1.v6.751

Badura, P., Madarasova, A., Geckova, M., Sigmund, E., Voracova, J., \& Sigmundova, D. (2016). Association between organized activity participation and healthy lifestyle in adolescents: Petr Badura. European Journal of Public Health, 26(1), 1. doi:10.1093/ eurpub/ckw170.041

Cava, M. J., Povedano, A., Buelga, S., \& Musitu, G. (2015). Análisis psicométrico de la Escala de Ajuste Escolar Percibido por el Profesor (PROF-A). Psychosocial Intervention, 24(2), 63-69. doi: 10.1016/j.psi.2015.04.001

Chok, N. S. (2010). Pearson's versus Spearman's and Kendall's correlation coefficients for continuous data (Tesis doctoral inédita). Universidad de Pittsburgh, Pensilvania.

Coelho, V. A., Marchante, M., \& Jimerson, S. R. (2017). Promoting a Positive Middle School Transition: A Randomized-Controlled Treatment Study Examining SelfConcept and Self-Esteem. Journal of Youth and Adolescence, 46(3), 558-569. doi:10.1007/ s10964-016-0510-6

Costa, S., \& Tabernero, C. (2012). Rendimiento académico y autoconcepto en estudiantes de educación secundaria obligatoria según el género. Revista Iberoamericana de Psicología y Salud, 3(2), 175-193. Recuperado de https://helvia.uco.es/xmlui/handle/10396/9281

Esnaola, I., Goñi, A., \& Madariaga, J. M. (2008). El autoconcepto: perspectivas de investigación. Revista de Psicodidáctica, 13(1), 179-194. Recuperado de http://www. redalyc.org/pdf/175/17513105.pdf 
Feldman, R. S. (2007). Desarrollo psicológico a través de la vida. México: Pearson Educación. Fernández-Zabala, A., Goñi, E., Rodríguez-Fernandez, A., \& Goñi A. (2015). Un nuevo cuestionario en castellano con escalas de las dimensiones del autoconcepto. Revista Mexicana de Psicología, 32(2), 149-159. Recuperado de http://www.redalyc.org/ $\mathrm{html} / 2430 / 243045364005 /$

Fernández-Zabala, A., \& Sarasa, M. (2015). El autoconcepto multidimensional. En A. Fernández-Zabala \& L. Revuelta (Eds.), Ajuste personal y social. Investigación psicoeducativa (pp. 59-73). Donostia: Erein.

Froiland, J. M., \& Davison, M. L. (2016). The longitudinal influences of peers, parents, motivation, and mathematics course-taking on high school math achievement. Learning and Individual Differences, 50, 252-259. doi:10.1016/j.lindif.2016.07.012

Fuentes, M. C., Alarcón, A., Gracia, E., \& García, F. (2015). El ajuste escolar en los adolescentes españoles: influencia de la socialización parental. Cultura y Educación, 27(1), 1-32. doi:10.1080/11356405.2015.1006847

Garaigordobil, M., Cruz, S., \& Pérez, J. I. (2003). Análisis correlacional y predictivo del autoconcepto con otros factores conductuales, cognitivos y emocionales de la personalidad durante la adolescencia. Estudios de Psicología, 24(1), 113-134. doi:10.1174/021093903321329102

Garaigordobil, M., Durá, A., \& Pérez, J. I. (2005). Psychopathological symptoms, behavioural problems, and selfconcept/self-esteem: A study of adolescents aged 14 to 17 years old. Annuary of Clinical and Health Psychology, 1, 53-63. Recuperado de http:// institucional.us.es/apcs/doc/APCS_1_eng_53-63.pdf

Garaigordobil, M., Pérez, J., \& Mozaz, M. (2008). Self-concept, self-esteem and psychopathological symptoms. Psicothema, 20(1), 114-123. Recuperado de http://www. redalyc.org/comocitar.oa?id $=72720116$

García, F., \& Musitu, G. (2009). AF5: Autoconcepto Forma 5. Madrid: TEA.

Gerard, J. M., \& Booth, M. Z. (2015). Family and school influences on adolescents' adjustment: The moderating role of youth hopefulness and aspirations for the future. Journal of Adolescence, 44, 1-16. doi:10.1016/j.adolescence.2015.06.003

González-Pienda, J. A., Fernández-Cueli, M., García, T., Suárez, N., Fernández, E., Tuero-Herrero, E., \& Da Silva, E. H. (2012). Diferencias de género en actitudes hacia las mataméticas en la enseñanza obligatoria. Revista Iberoamericana de Psicología y Salud, 3(1), 55-73. Recuperado de http://www.redalyc.org/pdf/2451/245122736004.pdf

Guay, F., Ratelle, C. F., Roy, A., \& Litalien, D. (2010). Academic self-concept, autonomous academic motivation, and academic achievement: Mediating and additive effects. Learning and Individual Differences, 20(6), 644-653. doi:10.1016/j.lindif.2010.08.001

Heras, J., \& Navarro, R. (2012). Ajuste escolar, soledad y conducta agresiva entre estudiantes de Educación Secundaria. Qurriculum: Revista de Teoría, Investigación y Práctica Educativa, 25, 105-124. Recuperado de https://riull.ull.es/xmlui/ handle/915/10673?show=full

Hernando, Á., Oliva, A., \& Pertegal, M. A. (2012). Variables familiares y rendimiento académico en la adolescencia. Estudios de Psicología, 33(1), 51-65. doi:10.1174/021093912799803791

Hernando, A., Oliva, A. \& Pertegal, M. A. (2013). Diferencias de género en los estilos de vida de los adolescentes. Psychosocial Intervention, 22(1), 15-23. doi:10.5093/in2013a3 
Huang, C. (2011). Self-concept and academic achievement: A meta-analysis of longitudinal relations. Journal of School Psychology, 49(5), 505-528. doi:10.1016/j.jsp.2011.07.001

Hysing, M., Harvey, A. G., Linton, S. J., Askeland, K. G., \& Sivertsen, B. (2016). Sleep and academic performance in later adolescence: results from a large population-based study. Journal of Sleep Research, 25(3), 318-324. doi:10.1111/jsr.12373

Ibabe, I. (2016). Academic failure and child-to-parent violence: family protective factors. Frontiers in Psychology, 7, 1-11. doi:10.3389/fpsyg.2016.01538

Ibarra-Aguirre, E., \& Jacobo-García, H. (2016). La evolución del autoconcepto académico en adolescentes. Revista Mexicana de Investigación Educativa, 21(68), 45-70. Recuperado de http://www.scielo.org.mx/pdf/rmie/v21n68/1405-6666-rmie-21-68-00045.pdf

Igbo, J. N., Onu, V. C., \& Obiyo, N. O. (2015). Impact of Gender Stereotype on Secondary School Students' Self-Concept and Academic Achievement. SAGE Open, 5(1), 1-10. doi:10.1177/2158244015573934

Kiuru, N., Aunola, K., Vuori, J., \& Nurmi, J. E. (2007). The role of peer groups in adolescents' educational expectations and school adjustment. Journal of Youth and Adolescence, 36(8), 995-1009. doi:10.1007/s10964-006-9118-6

Ladd, G. W. (1989). Children's social competence and social supports: Precursors of early school adjustment? En B. Schneider, G. Attili, J. Nadel \& R. Weissberg (Eds.), Social competence in developmental perspective (pp. 277-291). Holanda: Kluwer. doi:10.1007/978-94-009-2442-0

Ladd, G. W., Kochenderfer-Ladd, B., \& Rydell, A. M. (2011). Children's Interpersonal Skills and School-Based Relationships. En P. K. Smith, \& C. H. Hart (Eds.), The Wiley-Blackwell Handbook of Childhood Social Development (pp. 181-206). Oxford: Wiley-Blackwell. doi:10.1002/9781444390933.ch10

Lee, Y., \& Chung, H. (2016). A longitudinal study of developmental trajectories and predictors of school adjustment in early adolescence. Studies on Korean Youth, 27(2), 187- 214. doi:10.14816/sky.2016.27.2.187

Lee, J., \& Shute, V. J. (2010). Personal and social-contextual factors in K-12 academic performance: An integrative perspective on student learning. Educational Psychologist, 45(3), 185-202. doi:10.1080/00461520.2010.493471

Liu, W. C., \& Wang, C. K. J. (2005). Academic self-concept: A cross-sectional study of grade and gender differences in a Singapore secondary school. Asia Pacific Education Review, 6(1), 20-27. Recuperado de https://link.springer.com/article/10.1007/ BF03024964

Longobardi, C., Prino, L. E., Marengo, D., \& Settanni, M. (2016). Student-teacher relationships as a protective factor for school adjustment during the transition from middle to high school. Frontiers in Psychology, 7, 1-9. doi:10.3389/fpsyg.2016.01988

Malo, S., Bataller, S., Casas, F., Gras, M. E., \& González, M. (2011). Análisis psicométrico de la escala multidimensional de autoconcepto AF5 en una muestra de adolescentes y adultos de Cataluña. Psicothema, 23(4), 871-878. Recuperado de http://www. psicothema.com/psicothema.asp?id=3969

Marsh, H. W. (1986). Verbal and math self-concepts: An internal/external frame of reference model. American Educational Research Journal, 23(1), 129-149. doi: https:// doi.org/10.3102\%2F00028312023001129 
Marsh, H. W. (1990). The structure of academic self-concept: The Marsh/Shavelson model. Journal of Educational psychology, 82(4), 623-636. doi:10.1037/0022-0663.82.4.623

Marsh, H. W., Abduljabbar, A. S., Parker, P. D., Morin, A. J., Abdelfattah, F., Nagengast, B., ... Abu-Hilal, M. M. (2015). The internal/external frame of reference model of self-concept and achievement relations: Age-cohort and cross-cultural differences. American Educational Research Journal, 52(1), 168-202. doi:10.3102/0002831214549453

Marsh, H. W., Byrne, B. M., \& Shavelson, R. J. (1988). A multifaceted academic selfconcept: Its hierarchical structure and its relation to academic achievement. Journal of Educational Psychology, 80(3), 366-380. doi:10.1037/0022-0663.80.3.366

Marsh, H. W., Kuyper, H., Seaton, M., Parker, P. D., Morin, A. J., Möller, J., \& Abduljabbar, A. S. (2014). Dimensional comparison theory: An extension of the internal/ external frame of reference effect on academic self-concept formation. Contemporary Educational Psychology, 39(4), 326-341. doi:10.1016/j.cedpsych.2014.08.003

Marsh, H. W., \& Martin, A. J. (2011). Academic self-concept and academic achievement: Relations and causal ordering. British Journal of Educational Psychology, 81(1), 59-77. doi:10.1348/000709910X503501

Marsh, H. W., Smith, I. D., \& Barnes, J. (1985). Multidimensional self-concepts: Relationships with sex and academic achievement. Journal of Educational Psychology, 77(5), 581-596. doi:10.1037/0022-0663.77.5.581

Matalinares, M. L., Arenas, C., Dioses, A., Muratta, R., Pareja, C., \& Chávez, J. (2005). Inteligencia emocional y autoconcepto en colegiales de Lima Metropolitana. Revista IIPSI, 8(2), 41-55. doi: http://dx.doi.org/10.15381/rinvp.v8i2.4047

Montilla, J. M., \& Kromrey, J. (2010). Robustez de las pruebas T en comparación de medias, ante violación de supuestos de normalidad y homocedasticidad. Ciencia e Ingeniería, 31(2), 101-108. Recuperado de http://erevistas.saber.ula.ve/index.php/ cienciaeingenieria/article/view/1125

Moral de la Rubia, J.C., Sánchez-Sosa, J. C., \& Villarreal-González, M. E. (2010). Desarrollo de una escala multidimensional breve de ajuste escolar. REMA Revista Electrónica de Metodología Aplicada, 15(1), 1-11. doi: https://doi.org/10.17811/rema.15.1.2010.1-11

Mortimer, J. T., Zhang, L., Wu, C., Hussemann, J., \& Johnson, M. K. (2017). Familial transmission of educational plans and the academic self-concept: A three-generation longitudinal study. Social Psychology Quarterly, 80(1), 85-107. doi:10.1177/0190272516670582

Nunnally, J. C. (1976). Psychometric theory. Nueva York: McGraw-Hill.

Ramos-Díaz, E., Rodríguez-Fernández, A., Ros, I., \& Antonio-Agirre, I. (2017). Implicación escolar y autoconcepto multidimensional en una muestra de estudiantes españoles de secundaria. Revista Complutense de Educación, 28(4), 1103-1118. doi:10.5209/ RCED.51600

Rodríguez-Fernández, A., Droguett, L., \& Revuelta, L. (2012). Ajuste escolar y personal en la adolescencia: El papel del autoconcepto académico y del apoyo social percibido. Revista de Psicodidáctica, 17(2). doi:10.1387/Rev.Psicodidact.3002

Rodríguez-Fernández, A., Ramos-Díaz, E., Madariaga, J.M., Arribillaga, A., \& Galende, N. (2016). Steps in the construction and verification of an explanatory model of psychosocial adjustment. European Journal of Education and Psychology, 9(1), 20-28. doi: 10.1016/j.ejeps.2015.11.002 
Rodríguez-Fernández, A., Ramos-Díaz, E., Ros, I., Fernández-Zabala, A., \& Revuelta, L. (2016). Bienestar subjetivo en la adolescencia: el papel de la resiliencia, el autoconcepto y el apoyo social percibido. Suma Psicológica, 23(1), 60-69. doi:10.1016/j. sumpsi.2016.02.002

Shavelson, R. J., Hubner, J. J., \& Stanton, G. C. (1976). Self-concept: Validation of construct interpretations. Review of Educational Research, 46(3), 407-441. doi:10.2307/1170010

Stankov, L., \& Lee, J. (2014). Quest for the best non-cognitive predictor of academic achievement. Educational Psychology, 34(1), 1-8. doi:10.1080/01443410.2013.858908

Steinberg, L. (2005). Cognitive and affective development in adolescence. Trends in Cognitive Sciences, 9(2), 69-74. doi:10.1016/j.tics.2004.12.005

Suárez-Álvarez, J., Fernández-Alonso, R., \& Muñiz, J. (2014). Self-concept, motivation, expectations, and socioeconomic level as predictors of academic performance in mathematics. Learning and Individual Differences, 30, 118-123. doi:10.1016/j.lindif.2013.10.019

Veas, A., Gilar, \& Miñano, P. (2016). The Influence of Gender, Intellectual Ability, Academic Self-Concept, Self-Regulation, Learning Strategies, Popularity and Parent Involvement in Early Adolescence. International Journal of Information and Education Technology, 6(8), 591-597. doi:10.7763/IJIET.2016.V6.757

Vicent, M., Lagos-San Martín, N., Gonzálvez, C., Inglés, C. J., García-Fernández, J.M., \& Gomis, N. (2015). Diferencias de género y edad en autoconcepto en estudiantes adolescentes chilenos. Revista de Psicología, 24(1), 1-16. doi:10.5354/0719-0581.2015.36752

Wang, M. T., \& Fredricks, J. A. (2014). The reciprocal links between school engagement, youth problem behaviors, and school dropout during adolescence. Child Development, 85(2), 722-737. doi:10.1111/cdev.12138

Wentzel, K., Russell, S., \& Baker, S. (2014). Peer relationships and positive adjustment at school. En M. J. Furlong, R. Gilman \& E. S. Huebner (Eds.), Handbook of positive psychology in schools (2a ed., pp. 229-243). Oxford: Routledge.

West, S. G., Finch, J. F., \& Curran, P. J. (1995). Structural equation models with nonnormal variables: Problems and remedies. En R. H. Hoyle (Ed.), Structural equation modeling: Concepts, issues, and applications (pp. 56-75). Thousand Oaks, California: Sage Publications.

Fecha de recepción: 1 de noviembre de 2017.

Fecha de revisión: 20 de noviembre de 2017.

Fecha de aceptación: 17 de mayo de 2018. 
